\title{
Schedule-Dependent Effect of Epigallocatechin-3-Gallate (EGCG) with Paclitaxel on H460 Cells
}

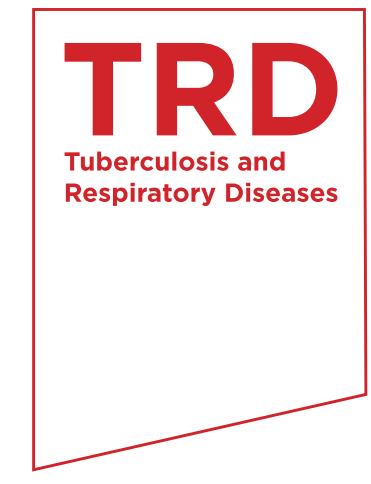

\author{
Sunghoon Park, M.D., Ph.D., Joo-Hee Kim, M.D., Yong Il Hwang, M.D., Ki-Suck Jung, M.D., Ph.D., \\ Young Sook Jang, M.S. and Seung Hun Jang, M.D., Ph.D. \\ Division of Pulmonary, Allergy and Critical Care Medicine, Department of Internal Medicine, Hallym University Sacred Heart \\ Hospital, Hallym University College of Medicine, Anyang, Korea
}

\begin{abstract}
Background: Epigallocatechin-3-gallate (EGCG), a major biologically active component of green tea, has anti-cancer activity in human and animal models. We investigated the schedule-dependent effect of EGCG and paclitaxel on growth of NCI-H460 non-small cell lung cancer cells.

Methods: To investigate the combined effect of EGCG (E) and paclitaxel (P), combination indices (CIs) were calculated, and cell cycle analysis was performed. For the effect on cell apoptosis, western blot analysis was also performed.

Results: CI analysis demonstrated that both concurrent and sequential $\mathrm{E} \rightarrow \mathrm{P}$ treatments had antagonistic effects (CIs $>1.0$ ), but sequential $\mathrm{P} \rightarrow \mathrm{E}$ had synergistic effects (CIs $<1.0$ ), on the growth inhibition of NCI-H460 cells. In the cell cycle analysis, although paclitaxel induced $\mathrm{G}_{2} / \mathrm{M}$ cell cycle arrest and increased the sub-G1 fraction, concurrent EGCG and paclitaxel treatments did not have any additive or synergistic effects compared with the paclitaxel treatment alone. However, western blot analysis demonstrated that sequential $\mathrm{P} \rightarrow \mathrm{E}$ treatment decreased the expression of Bcl-2 and procaspase-3 and increased poly(ADP-ribose) polymerase (PARP) cleavage; while minimal effects were seen with concurrent or sequential $\mathrm{E} \rightarrow \mathrm{P}$ treatments.

Conclusion: Concurrent or sequential $\mathrm{E} \rightarrow \mathrm{P}$ treatment had opposite effects to $\mathrm{P} \rightarrow \mathrm{E}$ treatment, where $\mathrm{P} \rightarrow \mathrm{E}$ treatment showed a synergistic effect on growth inhibition of NCI-H460 cells by inducing apoptosis. Thus, the efficacy of EGCG and paclitaxel combination treatment seems to be schedule-dependent.
\end{abstract}

Keywords: Epigallocatechin Gallate; Lung Neoplasms; Paclitaxel; Cell Cycle

Address for correspondence: Seung Hun Jang, M.D., Ph.D.

Division of Pulmonary, Allergy and Critical Care Medicine, Department of Internal Medicine, Hallym University Sacred Heart Hospital, Hallym University College of Medicine, 22 Gwanpyeong-ro 170beon-gil,

Dongan-gu, Anyang 431-796, Korea

Phone: 82-31-380-3718, Fax: 82-31-380-3973

E-mail: chestor@hallym.or.kr

Received: Dec. 7, 2013

Revised: Jan. 9, 2014

Accepted: Jan. 21, 2014

(c) It is identical to the Creative Commons Attribution Non-Commercial License (http://creativecommons.org/licenses/by-nc/3.0/).

Copyright (c) 2014

The Korean Academy of Tuberculosis and Respiratory Diseases. All rights reserved.

\section{Introduction}

Anti-cancer effects of green tea preparations have been demonstrated in many cancer cell lines and animal models ${ }^{1-3}$, suggesting that green tea may be a cancer preventative. The primary anti-cancer components of green tea are the catechins and epigallocatechin-3-gallate (EGCG) is one of the catechins with the largest biological activity and most frequently demonstrated anti-cancer activity in human and animal models for lung, skin, colon, prostate, and breast cancers ${ }^{3-7}$.

The biological activities of EGCG include anti-oxidant and anti-inflammatory functions, inhibition of angiogenesis and cancer invasion, and induction of cell cycle arrest and apoptosis ${ }^{8}$. EGCG can also inhibit telomerase function, which is a target of chemotherapy. However, despite positive results 
from epidemiological and experimental studies, the effect of EGCG in oncologic practice is not clear. The peak blood level by maximal injection cannot reach inhibitory cancer cell levels in the human body, and a phase I study showed that green tea extract alone was not an effective anti-cancer agent ${ }^{9,10}$.

Paclitaxel is a member of the taxane family and causes the stabilization of mitotic spindle microtubules by binding the $\beta$-tubulin subunit, which leads to $\mathrm{G}_{2} / \mathrm{M}$ cell cycle arrest and apoptosis $^{11,12}$. Recent studies of paclitaxel combination treatments have demonstrated that sequential regimens can have a synergistic effect on cancer cell lines ${ }^{13-15}$. We hypothesized that even though EGCG itself may not have any anti-cancer activity, a combination of EGCG with other proven chemotherapeutic agents would have a synergistic effect and that different schedules would differentially affect cancer cells. Therefore, in this study, we investigated the effect of concurrent and sequential combination treatments with EGCG and paclitaxel on growth of NCI-H460, a human non-small cell lung cancer (NSCLC) cell line.

\section{Materials and Methods}

\section{Cell culture and growth curves}

NCI-H460 cells were cultured and maintained in RPMI1640 media (Sigma Chemical Co., St Louis, MO, USA) supplemented with $10 \%$ inactivated fetal bovine serum (FBS) (Sigma Chemical Co.), $100 \mathrm{U} / \mathrm{mL}$ penicillin $\mathrm{G}$ and $10 \mu \mathrm{g} / \mathrm{mL}$ streptomycin in humidified incubator at $37^{\circ} \mathrm{C}$ with $5 \% \mathrm{CO}_{2}$. Viable cell growth was analyzed using a 3-[4.5-dimethylthiazol-2-yl]2,5-diphenyltetrazoliumbromide (MTT) assay kit ${ }^{16}$. The cell seeding number was determined from the exponential growth area derived from cell growth curves.

\section{Cell growth inhibition}

Harvested H460 cells were plated at a density of 5,000/well in a 96-well tissue culture plate. After overnight incubation, cells were treated with various concentrations of EGCG and paclitaxel in RPMI-1640 with 10\% FBS for 72 hours. Following a 72-hour treatment with each agent, cell proliferation assays were performed using a MTT assay kit according to the manufacturer's instructions. The drug concentrations inhibiting cell growth by $50 \%\left(\mathrm{IC}_{50}\right)$ were determined by interpolation from the dose-response curves. Each point represents the mean \pm standard deviation (SD) of triplicate wells.

\section{Combination index assay}

The combination index (CI) was calculated to investigate the combined effect of EGCG and paclitaxel. NCI-H460 cells were seeded in 96 -well plates at a density of 5,000/well over- night prior to drug treatment. After overnight incubation, the cells were concurrently treated for 72 hours with EGCG and paclitaxel (i.e., E+P), and growth inhibition was measured by MTT assay. For the sequential treatment, after overnight incubation the cells were treated with EGCG first (24 hours), and then were treated with paclitaxel (24 hours) after removal of the media containing EGCG. Finally, they were incubated for an additional 24 hours (i.e., $\mathrm{E} \rightarrow \mathrm{P}$ ). Growth inhibition was assessed by MTT assay, and the same procedure with the reverse sequence was also performed (i.e., $\mathrm{P} \rightarrow \mathrm{E}$ ). Each drug combination was tested at four different concentrations, and the CI calculated using a formula reported previously; $\mathrm{CI}>1$ was defined as an antagonistic effect, $\mathrm{CI}=1$ as an additive effect, and $\mathrm{CI}<1$ as a synergistic effect ${ }^{17}$.

\section{Cell cycle analyses}

Cell suspensions were plated on 60-mm culture plates and incubated overnight. They were treated with $150 \mu \mathrm{M}$ EGCG alone, $10 \mathrm{nM}$ paclitaxel alone and their combinations $(\mathrm{E}+\mathrm{P})$ for 24 hours. The cells were washed using phosphate-buffered saline (PBS) and fixed with 70\% ethanol for 1 hour, and then washed again with PBS and stained with propidium iodide (Molecular Probes, Eugene, OR, USA). Cell cycle analyses were performed using FACScan (Becton Dickinson, San Jose, CA, USA).

\section{Bcl-2, procaspase-3, and PARP expression}

Western blotting for Bcl-2, procaspase-3, and poly(ADPribose) polymerase (PARP) cleavage were performed to investigate the effect of EGCG and paclitaxel on cell apoptosis. After a 24-hour incubation, cells were treated with $150 \mu \mathrm{M}$ EGCG and $10 \mathrm{nM}$ paclitaxel concurrently for an additional 24 hours $(\mathrm{E}+\mathrm{P})$. In addition, to see the effect of sequential treatments, cells were sequentially treated for 24 hours with each drug (E $\rightarrow \mathrm{P}$ and $\mathrm{P} \rightarrow \mathrm{E}$ ) after the initial 24-hour incubation.

For protein extraction, cells were washed with PBS and lysed in a RIPA buffer containing protease inhibitors. These lysates were centrifuged at $4^{\circ} \mathrm{C}$ for 20 minutes at $14,000 \mathrm{rpm}$ and supernatants containing protein collected. Protein concentrations were determined using the Bio-Rad Protein Assay kit (Bio-Rad, Hercules, CA, USA). A total of $30 \mu \mathrm{g}$ of protein was separated by sodium dodecyl sulfate polyacrylamide gel electrophoresis. After transfer to a polyvinylidene fluoride membrane, the blots were blocked with 5\% fat free milk in TBS-T (20 mM Tris-HCl, pH 7.4, containing $150 \mathrm{mM} \mathrm{NaCl}$ and $0.05 \%$ Tween 20$)$ and incubated with primary antibodies (1:200, Bcl-2, Santa Cruz Biotechnology Inc., Santa Cruz, CA, USA; 1:200, procaspase-3, Santa Cruz Biotechnology Inc.; and 1:5,000, PARP, Cell Signaling Technology Inc., Danvers, MA, USA) overnight at $4^{\circ} \mathrm{C}$. After washing, blots were incubated with horseradish peroxidase-conjugated secondary 
antibodies (Santa Cruz Biotechnology Inc.) for 1 hour at room temperature. The protein bands were visualized using an enhanced chemiluminescence kit (GE Healthcare, Little Chalfont, UK).

\section{Statistical analysis}

Continuous data are presented as means $\pm \mathrm{SD}$ and the statistical differences calculated using a student t-test. A $p<0.05$ was considered to indicate statistical significance. All data shown are representative of three independent experiments.

\section{Results}

1. EGCG and paclitaxel inhibit the growth of NSCLC cell lines

The growth of NCI-H460 cells was inhibited by EGCG and paclitaxel in a dose-dependent pattern (Figure 1). The $\mathrm{IC}_{50} \mathrm{val}-$ ues of EGCG and paclitaxel were $150 \mu \mathrm{M}$ and $5 \mathrm{nM}$, respectively.

2. Combination indices show that sequential paclitaxel $\rightarrow$ EGCG treatments cause synergistic inhibition of NCI-H460 cell growth

NCI-H460 cells were treated with various concentrations of EGCG $(75,100,125$, and $150 \mu \mathrm{M})$ and paclitaxel $(1,2.5,5$, and $7.5 \mathrm{nM}$ ) concurrently or sequentially, and growth inhibition curves plotted. The CIs of different concentrations were calculated from the curves (Figure 2). Both concurrent and sequential $\mathrm{E} \rightarrow \mathrm{P}$ treatments showed antagonistic effects $(\mathrm{CI}>1.0)$ as the dose of each drug increased. However, sequential $\mathrm{P} \rightarrow \mathrm{E}$ treatments showed synergistic effects $(\mathrm{CI}<1.0)$ when the pa- clitaxel concentration was $>5 \mathrm{nM}$.

3. EGCG does not have a synergistic effect on cell cycle arrest when concurrently added to paclitaxel treatment

The distribution of cells in different phases of the cell cycle is shown in Figure 3. Treatment with EGCG alone showed a significant but minimal increase in the sub- $G_{1}$ fraction $(\mathrm{p}=0.042)$. In contrast, treatment with paclitaxel alone markedly induced $\mathrm{G}_{2} / \mathrm{M}$ cell cycle arrest $(\mathrm{p}=0.001)$ and increased the sub- $\mathrm{G}_{1}$ fraction $(\mathrm{p}=0.003)$. Concurrent $(\mathrm{E}+\mathrm{P})$ treatment showed similar results to paclitaxel alone with cell cycle arrest in $\mathrm{G}_{2} / \mathrm{M}$. Therefore, EGCG did not seem to have an additive or synergistic effect on cell cycle arrest when added to paclitaxel treatment.

\section{Sequential paclitaxel $\rightarrow$ EGCG treatments improved induction of apoptosis}

Western blotting for Bcl-2, procaspase-3, and PARP cleavage was performed with NCI-H460 cells treated with EGCG and paclitaxel (Figure 4). The inhibitory effect was not significant when the cells were treated with both drugs concurrently. However, sequential $\mathrm{P} \rightarrow \mathrm{E}$ treatment significantly inhibited the expression of Bcl-2, which was not observed with $\mathrm{E} \rightarrow \mathrm{P}$ treatment. Furthermore, Sequential $\mathrm{E} \rightarrow \mathrm{P}$ and $\mathrm{P} \rightarrow \mathrm{E}$ treatments reduced the expression of procaspase-3, indicating caspase- 3 activation, while concurrent treatments showed a borderline effect. In particular, the effect of sequential $\mathrm{P} \rightarrow$ $\mathrm{E}$ treatment was significantly greater than that of $\mathrm{E} \rightarrow \mathrm{P}$ treatment. Sequential $P \rightarrow E$ also significantly increased the expression of cleaved PARP activity, compared to other treatment modalities.

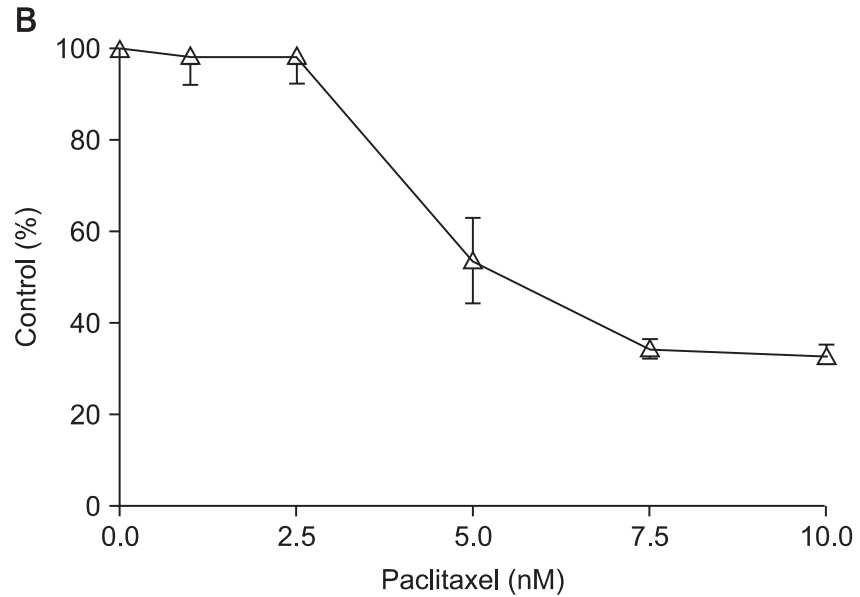

Figure 1. Growth inhibition curves. The growth of NCI-H460 cells was inhibited by epigallocatechin-3-gallate (EGCG) (A) and paclitaxel (B) in a dose-dependent manner. 
A
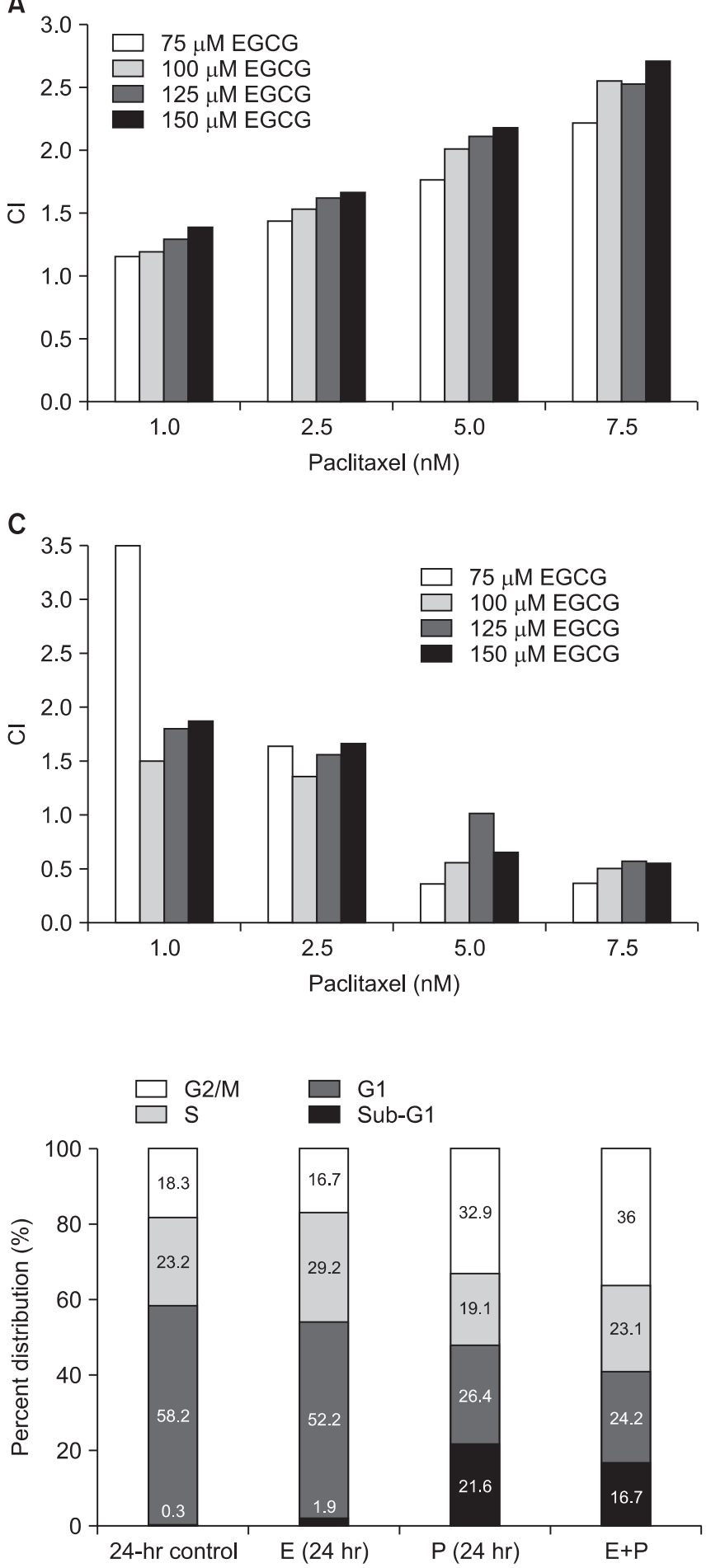

Figure 3. Cell cycle analysis. EGCG treatment alone slightly increased the sub- $\mathrm{G}_{1}$ fraction ( $\mathrm{p}=0.042$ vs. control). In contrast, paclitaxel induced $\mathrm{G}_{2} / \mathrm{M}$ cell cycle arrest ( $\mathrm{p}=0.001$ vs. control) and increased the sub- $\mathrm{G}_{1}$ fraction ( $\mathrm{p}=0.003$ vs. control). When combined, concurrent $(\mathrm{E}+\mathrm{P})$ treatment showed similar results to paclitaxel alone. E: epigallocatechin-3-gallate (EGCG); P: paclitaxel.

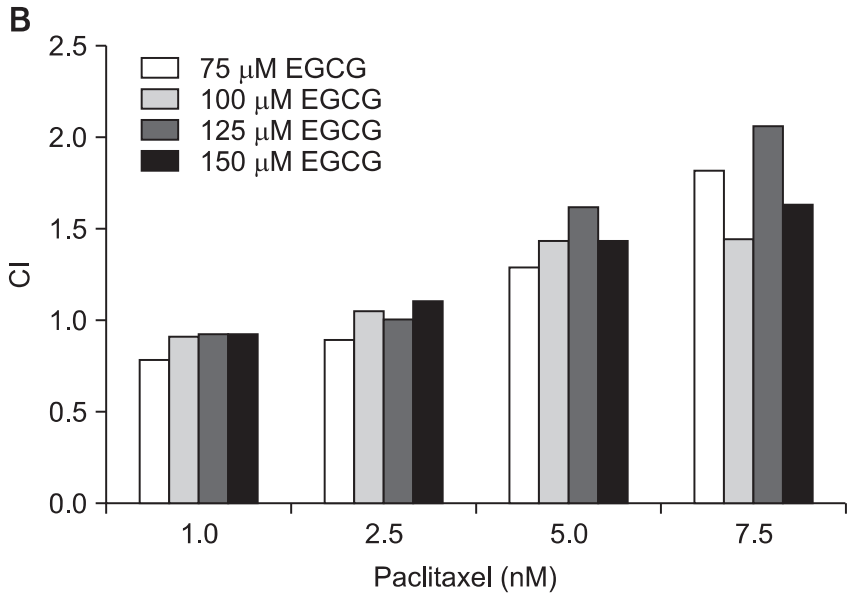

Figure 2. Combination indices (CIs). Both concurrent (A) and sequential $\mathrm{E} \rightarrow \mathrm{P}(\mathrm{B})$ treatments showed dose-dependent antagonistic effects (CI>1.0) on NCI-H460 cells. However, sequential $\mathrm{P} \rightarrow$ E treatments (C) showed synergistic effects $(\mathrm{CI}<1.0)$ when the paclitaxel concentration was greater than $5 \mathrm{nM}$. E: epigallocatechin-3-gallate (EGCG); P: paclitaxel.

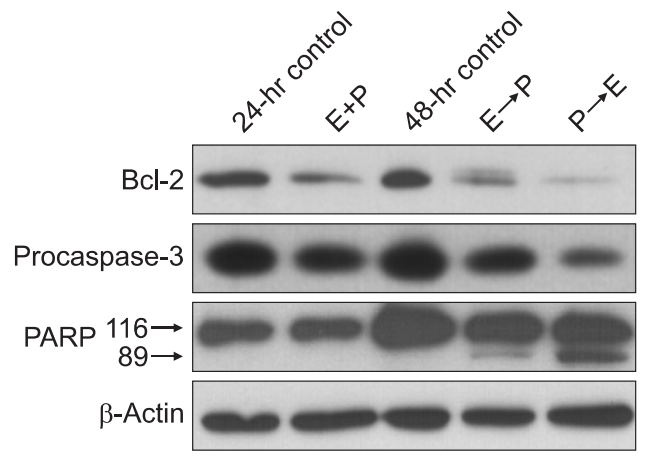

Figure 4. Expressions of Bcl-2, procaspase-3, and cleaved poly(ADPribose) polymerase-89 (PARP-89). Sequential $\mathrm{P} \rightarrow \mathrm{E}$ treatment decreased the expression of $\mathrm{Bcl}-2$ and procaspase- 3 , and increased the expression of cleaved PARP-89. Sequential E $\rightarrow$ P treatment also decreased the expression of procaspase- 3 to a lesser extent than sequential $\mathrm{P} \rightarrow$ E treatment. E: epigallocatechin-3-gallate $(150 \mu \mathrm{M})$; P: paclitaxel (10 $\mathrm{nM})$. 


\section{Discussion}

This study demonstrated that sequential $\mathrm{P} \rightarrow \mathrm{E}$ treatment had a synergistic effect on inhibition of NCI-H460 growth when the concentration of paclitaxel was $>5 \mathrm{nM}$. Additionally, EGCG treatment did not have any additive or synergistic effect on cell cycle arrest when concurrently added to paclitaxel. Moreover, western blotting results indicate that sequential $\mathrm{P} \rightarrow$ E treatment can be more effective in the induction of apoptosis, compared to other combination modalities.

There are many studies describing the effect of EGCG on cancer cells. Nihal et al. ${ }^{18}$ demonstrated that EGCG induced apoptosis in melanoma cells by inhibiting the expression of Bcl-2, increasing the expression of Bax, caspase-3, caspase-7, and caspase- 9 , and causing $S$ phase and $G_{1}$ phase arrest. In colorectal cancer cells, Inaba et al. ${ }^{19}$ showed that, although EGCG increased the phosphorylation of JNK1/2 and ERK1/2 mitogen-activated protein kinases (MAPKs) and increased the expression of caspase-3 and PARP, it inhibited the expression of cyclin $\mathrm{D}_{1}$ and did not affect the cell cycle. This suggested that the effect of EGCG on cancer cells might be associated with MAPK-mediated apoptosis rather than regulation of the cell cycle. Sadava et al. ${ }^{20}$ investigated the effect of EGCG on small cell lung cancer cells and reported that EGCG induced apoptosis by inhibiting telomerase activity, increasing the expression of caspase- 3 and caspase- 9 , and causing $S$ phase arrest. In NCI-H460 NSCLC cells, our study has demonstrated that EGCG did not have a significant impact on cell cycle arrest, contrary to previous reports. However, the effects of EGCG on the cell cycle can be cell-line dependent and epidemiologic evidence describing the effect of green tea consumption on cancer is conflicting ${ }^{19-22}$. Therefore, further studies, including clinical research, are needed to clarify the definite mechanisms and roles of EGCG on cancer cells.

Paclitaxel belongs to the taxane family, which stabilizes microtubules by binding to $\beta$-tubulin. The strong inhibitory effect of paclitaxel on lung cancer cells has been well documented; it causes $\mathrm{G}_{2} / \mathrm{M}$ cell cycle arrest and induces apoptosis by increasing the expression of caspase and decreasing the expression of Bcl-2. Our study confirmed these findings, demonstrating paclitaxel-induced $\mathrm{G}_{2} / \mathrm{M}$ arrest, decreased expression of Bcl-2 and procaspase-3, and increased PARP cleavage.

While combination treatments are widely used in the treatment of NSCLC, many in vitro studies have determined that their effects vary depending on the sequence in which the drugs are administered. In a study by Shimoyama et al. ${ }^{23}$, concurrent treatment of gefitinib and camptothecin showed an antagonistic effect on lung cancer cells, while a sequential treatment with camptothecin followed by gefitinib showed a synergistic effect, demonstrated by $\mathrm{S}$ phase arrest and increased apoptosis. In a study of paclitaxel, Cheng et al. ${ }^{13}$ also demonstrated when treating cancer cells sequentially with gefitinib followed by paclitaxel, gefitinib induced $G_{1}$ phase arrest and as a result, the paclitaxel did not have a sufficient cytotoxic effect. However, when treating cells in the reverse sequence, paclitaxel induced phosphorylation of epidermal growth factor receptor (EGFR) in mitotic cells, which increased the inhibitory effects of subsequent gefitinib treatment. However, several authors suggested that EGCG could also be useful in combination with conventional chemotherapeutics for the prevention and treatment of cancers because it is a non-toxic agent with potential for cancer prevention ${ }^{24}$. Zhang et al. ${ }^{25}$ studied the combination effect of EGCG with erlotinib and showed a synergistic effect on squamous cell carcinoma of the head and neck, in which EGCG induced EGFR internalization and ubiquitin-degradation.

In the present study of paclitaxel and ECGC, we found that concurrent treatment $(\mathrm{E}+\mathrm{P})$ showed an antagonistic effect (CI>1.0) on growth inhibition but had no effect on apoptosis. This indicates that the effect of concurrent treatment might be inferior to paclitaxel alone and that EGCG could diminish the efficacy of paclitaxel when administered concurrently. However, when EGCG was administered after paclitaxel (i.e., $\mathrm{P} \rightarrow \mathrm{E}$ ), a synergistic effect was observed based on the CIs and activation of apoptotic cell death was also observed, as demonstrated by decreased expression of Bcl-2 and procaspase-3 and increased PARP cleavage. These results suggest that when adding EGCG to conventional chemotherapy, a sequential treatment model should be considered.

However, in the present study, EGCG inhibited the growth of $\mathrm{H} 460$ cells but did not have a significant effect on cell cycle arrest and apoptotic cell death, suggesting that EGCG might inhibit cell growth via different mechanisms. Additionally, we could not demonstrate the exact mechanism that only sequential $\mathrm{P} \rightarrow \mathrm{E}$ treatment, not concurrent or sequential $\mathrm{E}$ $\rightarrow$ P models, showed a synergistic effect. Although these can be limitations of the present study, we think that the effects of EGCG can vary depending on the drug concentration and cancer cell line used and that the effect of combination treatments can be schedule-, as well as, time-dependent ${ }^{15}$. However, we did not vary drug concentration in western blotting nor did we investigate the time-dependent effect in the present study. Therefore, future studies with more detailed methods should be conducted to demonstrate the exact mechanisms.

Our results indicate that the chemotherapeutic effect can be maximized if sequential treatment is used when adding EGCG to other conventional agents. As there are few studies describing the efficacy of EGCG combination treatment with other chemotherapeutics and green tea is a non-toxic anticancer agent, the hypothesis tested in this study is relevant. However, before green tea can be used widely in clinical practice, more studies are needed to clarify the molecular interactions between EGCG and other chemotherapeutic agents. 


\section{Acknowledgements}

This study was supported by a grant (01-2004-22) from Hallym University Medical Center Research Fund.

\section{References}

1. Imai K, Suga K, Nakachi K. Cancer-preventive effects of drinking green tea among a Japanese population. Prev Med 1997; 26:769-75.

2. Ji BT, Chow WH, Hsing AW, McLaughlin JK, Dai Q, Gao YT, et al. Green tea consumption and the risk of pancreatic and colorectal cancers. Int J Cancer 1997;70:255-8.

3. Kato I, Tominaga S, Matsuura A, Yoshii Y, Shirai M, Kobayashi S. A comparative case-control study of colorectal cancer and adenoma. Jpn J Cancer Res 1990;81:1101-8.

4. Gao YT, McLaughlin JK, Blot WJ, Ji BT, Dai Q, Fraumeni JF Jr. Reduced risk of esophageal cancer associated with green tea consumption. J Natl Cancer Inst 1994;86:855-8.

5. Jankun J, Selman SH, Swiercz R, Skrzypczak-Jankun E. Why drinking green tea could prevent cancer. Nature 1997;387:561.

6. Komori A, Yatsunami J, Okabe S, Abe S, Hara K, Suganuma M, et al. Anticarcinogenic activity of green tea polyphenols. Jpn J Clin Oncol 1993;23:186-90.

7. Yang CS, Wang ZY. Tea and cancer. J Natl Cancer Inst 1993;85:1038-49.

8. Park OJ, Surh YJ. Chemopreventive potential of epigallocatechin gallate and genistein: evidence from epidemiological and laboratory studies. Toxicol Lett 2004;150:43-56.

9. Lambert JD, Yang CS. Mechanisms of cancer prevention by tea constituents. J Nutr 2003;133:3262S-7S.

10. Laurie SA, Miller VA, Grant SC, Kris MG, Ng KK. Phase I study of green tea extract in patients with advanced lung cancer. Cancer Chemother Pharmacol 2005;55:33-8.

11. Blagosklonny MV, Fojo T. Molecular effects of paclitaxel: myths and reality (a critical review). Int J Cancer 1999;83:1516.

12. Dumontet C, Sikic BI. Mechanisms of action of and resistance to antitubulin agents: microtubule dynamics, drug transport, and cell death. J Clin Oncol 1999;17:1061-70.

13. Cheng H, An SJ, Zhang XC, Dong S, Zhang YF, Chen ZH, et al. In vitro sequence-dependent synergism between paclitaxel and gefitinib in human lung cancer cell lines. Cancer Chemother Pharmacol 2011;67:637-46.

14. Oliveras-Ferraros C, Vazquez-Martin A, Colomer R, De Llorens R, Brunet J, Menendez JA. Sequence-dependent syner- gism and antagonism between paclitaxel and gemcitabine in breast cancer cells: the importance of scheduling. Int J Oncol 2008;32:113-20.

15. Zoli W, Ricotti L, Barzanti F, Dal Susino M, Frassineti GL, Milandri C, et al. Schedule-dependent interaction of doxorubicin, paclitaxel and gemcitabine in human breast cancer cell lines. Int J Cancer 1999;80:413-6.

16. Kano Y, Sakamoto S, Kasahara T, Akutsu M, Inoue Y, Miura Y. Effects of amsacrine in combination with other anticancer agents in human acute lymphoblastic leukemia cells in culture. Leuk Res 1991;15:1059-66.

17. Zhao L, Wientjes MG, Au JL. Evaluation of combination chemotherapy: integration of nonlinear regression, curve shift, isobologram, and combination index analyses. Clin Cancer Res 2004;10:7994-8004.

18. Nihal M, Ahmad N, Mukhtar H, Wood GS. Anti-proliferative and proapoptotic effects of (-)-epigallocatechin-3-gallate on human melanoma: possible implications for the chemoprevention of melanoma. Int J Cancer 2005;114:513-21.

19. Inaba H, Nagaoka Y, Kushima Y, Kumagai A, Matsumoto Y, Sakaguchi M, et al. Comparative examination of anti-proliferative activities of (-)-epigallocatechin gallate and (--)-epigallocatechin against HCT116 colorectal carcinoma cells. Biol Pharm Bull 2008;31:79-84.

20. Sadava D, Whitlock E, Kane SE. The green tea polyphenol, epigallocatechin-3-gallate inhibits telomerase and induces apoptosis in drug-resistant lung cancer cells. Biochem Biophys Res Commun 2007;360:233-7.

21. Higdon JV, Frei B. Tea catechins and polyphenols: health effects, metabolism, and antioxidant functions. Crit Rev Food Sci Nutr 2003;43:89-143.

22. Huh SW, Bae SM, Kim YW, Lee JM, Namkoong SE, Lee IP, et al. Anticancer effects of (-)-epigallocatechin-3-gallate on ovarian carcinoma cell lines. Gynecol Oncol 2004;94:760-8.

23. Shimoyama T, Koizumi F, Fukumoto H, Kiura K, Tanimoto M, Saijo N, et al. Effects of different combinations of gefitinib and irinotecan in lung cancer cell lines expressing wild or deletional EGFR. Lung Cancer 2006;53:13-21.

24. Singh BN, Shankar S, Srivastava RK. Green tea catechin, epigallocatechin-3-gallate (EGCG): mechanisms, perspectives and clinical applications. Biochem Pharmacol 2011;82:180721.

25. Zhang X, Zhang H, Tighiouart M, Lee JE, Shin HJ, Khuri FR, et al. Synergistic inhibition of head and neck tumor growth by green tea (-)-epigallocatechin-3-gallate and EGFR tyrosine kinase inhibitor. Int J Cancer 2008;123:1005-14. 